\title{
Erratum zu: Operationen am Kniegelenk in der Neuroorthopädie
}

Domenic Grisch und Thomas Dreher

Die aktualisierte Version des Kapitel 35 dieses Buches finden Sie unter

https://doi.org/10.1007/978-3-662-61330-6_35 


\section{Erratum zu:}

Kapitel 35 in: W. Strobl et al. (Hrsg.), Neuroorthopädie - Disability Management

- https://doi.org/

10.1007/978-3-662-61330-6_35

In einigen Abbildungen von Kapitel 35 wurden leider folgende Quellenhinweise vergessen

Abb. 35.7b

Ergänzung in Bildunterschrift:

(Kay 2015, mit freundlicher Genehmigung von Wolters Kluwer Health)
Abb. 35.7c

Ergänzung in Bildunterschrift:

(Nazareth 2020, mit freundlicher Genehmigung von Wolters Kluwer Health)

Abb. 35.8

Ergänzung in Bildunterschrift:

(Sossai 2015, mit freundlicher Genehmigung von Elsevier)

Abb. 35.11

Ergänzung in Bildunterschrift:

(Novacheck 2009, mit freundlicher Genehmigung von Wolters Kluwer Health)

Das wurde nun korrigiert. 